Cite this: Phys. Chem Chem. Phys, 2013, 15, 9219

Received 29th November 2012, Accepted 15th April 2013

DOI: $10.1039 / c 3 c p 44279 a$

www.rsc.org/pccp

\section{Analysis of the molecular interactions governing the polymorphism of benzamide - a guide to syntheses?}

\author{
Philipp Ectors and Dirk Zahn*
}

\begin{abstract}
Dispersion-corrected density functional calculations are used to rationalize the subtle differences in the molecular interactions in benzamide crystals. The potential energy of the different polymorph structures is dominated by the interplay between intermolecular attraction and molecular torsion/deformation to accommodate favourable molecular packing. Using suitable proxies arranged in pseudo-crystalline setups we discriminate the contribution of hydrogen bonding, $\pi-\pi$ interactions and intra-molecular interactions to the lattice energies of the most relevant (P1 and P3) benzamide polymorphs. Strikingly, these commonly anticipated binding/packing concepts cannot account for the observed ranking of benzamide structures, thus hinting at the importance of the interactions between the benzene rings and the polar amide groups. Moreover, individual structural motifs that account for the competition between the two crystal structure types are elaborated. On the basis of such in-depth understanding of molecular interactions - in terms of both structure and chemistry - we suggest nucleation scenarios as guides to a more controlled synthesis of the stable P1 form or to direct nucleation in favour of the P3 polymorph.
\end{abstract}

\section{Introduction}

Polymorphism in molecular crystals is an important issue, particularly for the pharmaceutical industry where most production routes aim at the solid state. The structure of molecular crystals is crucial in controlling parameters such as solubility, stability and metabolism of orally administered drugs. Indeed, druggability of a candidate molecule (here we refer to its suitability as a drug, following the definition in ref. 1) can entirely depend on its polymorphism: very stable polymorphs tend to be less soluble giving rise to problems during the liberation process of the drug, whilst less stable polymorphs might be too easily soluble (or worse transform before application) and also turn out a failure. Thus, the stability problem is encountered twice, unwanted phase transitions may occur when applying pressure during the pelleting process and poorly soluble polymorphs fail to pass the intestinal membrane. Long term stability also constitutes a crucial issue for drugs that require storage over years. Here, even very slow transformation processes can spoil the success of a drug candidate.

For these reasons, considerable efforts are being invested in finding and ranking new polymorphs. While structure identification from experiments is still most common, rapid progress in theory has paved the way for suggesting structures and ranking

Lehrstuhl für Theoretische Chemie/Computer-Chemie-Centrum Friedrich-AlexanderUniversität Erlangen-Nürnberg, Nägelsbach Str. 25, D-91052 Erlangen, Germany.

E-mail:dirk.zahn@chemie.uni-erlangen.de them in terms of energy and/or enthalpy..$^{2-7}$ Moreover, molecular dynamics simulations, if combined with accurate force-fields may provide insights into polymorphic transformations ${ }^{8-10}$ and into crystal nucleation. ${ }^{11}$ While characterization of such process dynamics in most cases needs to rely on force-field models to avoid outrageous computational costs, for well-chosen structural motifs we may rationalize the underlying interactions using more accurate quantum calculations.

In what follows, we outline such calculations suing the example of benzamide crystals. This system has evolved to a common proxy to molecular crystal polymorphism, with experimental experience dating back to Friedrich Wöhler and Justus von Liebig in $1832 .^{12}$ Using state-of-the-art density functional calculations, we shall not only investigate structure candidates (P1 and polymorphs P2, P3) for the benzamide crystal itself, but also construct artificial 'crystals' comprising fragments of benzamide and acting as proxies for selected interaction types. Thus, we probe whether different types of molecular interactions can be 'isolated' from a complex interplay of various contributions in a molecular crystal. Such in-depth characterization in terms of interaction models could boost the molecular scale understanding of polymorphism and thus provide guides to the rationalized design of synthesis routes.

\section{Calculation details}

Density-functional theory (DFT) calculations ${ }^{13}$ were performed using the Perdew-Burke-Ernzerhof exchange correlation functional, ${ }^{14}$ 
ultrasoft pseudopotentials with a plane wave cutoff of 35 Ry and a secondary cutoff of 180 Ry along with an empirical dispersion correction as proposed by Grimme. ${ }^{15}$ For the (pseudo)crystal structure calculations, periodic boundary conditions are applied to unit cell models comprising 4 (P1 and P3 structures) and 8 (P2) explicit molecules, respectively. ${ }^{16-18}$ Accordingly, a Monkhorst $k$-point grid $^{19}$ of $4 \times 4 \times 1$ and $1 \times 1 \times 3$ was found to be appropriate for the $\mathrm{P} 1 / \mathrm{P} 3$ and $\mathrm{P} 2$ structures, respectively.

Energies of crystal formation were calculated with respect to isolated molecules. For this a single benzamide molecule was placed in a pseudo-crystal of sufficiently large unit cell (corresponding to a $3 \times 3 \times 1$ supercell) to avoid intermolecular interactions from periodic boundary conditions. As proxies to investigate hydrogen bonding and $\pi-\pi$-interactions on an exclusive basis, we prepared in principle artificial arrangements of benzene and formamide molecular crystals, based on the crystal structures of benzamide. For this, the atomic positions of the corresponding fragment of benzamide $\left(\mathrm{C}_{6} \mathrm{H}_{5}-\right.$ and $\left.-\mathrm{CONH}_{2}\right)$ were frozen, whilst replacing the remaining part $\left(\mathrm{C}_{6} \mathrm{H}_{5}-\right.$ and $\left.-\mathrm{CONH}_{2}\right)$ by a hydrogen atom to obtain molecules. Upon relaxation of the newly formed $\mathrm{C}-\mathrm{H}$ bond whilst fixing the rest of the system, we thus construct benzene and formamide molecular crystals that are artificial by design, but directly correspond to the crystal structures of benzamide polymorphs and thus aim at mimicking specific interactions therein.

\section{Results}

Dispersion-corrected DFT calculations were employed to optimize the crystal structure of the three known forms of benzamide, named P1 (commonly considered as the stable form), P2 (very unstable, cannot be synthesized in pure form so far) and P3 (recently grown as single crystals ${ }^{17}$ ). Therein, full structure relaxation including unit cell size and shape is performed. The structural parameters as obtained from potential energy minimization are shown in Table 1. While P2 - which is known to be rather unstable - displays the least favourable formation energy, the two polymorphs P1 and P3 differ by only $1.9 \mathrm{~kJ} \mathrm{~mol}^{-1}$ (per unit cell).

It is worthwhile to contrast the two most important forms of benzamide crystals, i.e. the stable P1 form and the polymorph P3 in terms of structural motifs and molecular interactions therein.
The commonly considered concepts for rationalizing the energetic ranking of benzamide polymorphs rely on hydrogen bonding and its competition with benzene-benzene interactions. ${ }^{17,20}$ While these chemical bonding/molecular packing concepts are not separable from a DFT calculation yielding only the total energy of crystal formation, we designed artificial molecular crystals to provide a closer look focussing on individual contributions. Indeed, using benzene and formamide as proxies to investigate the $\pi-\pi$ interactions and hydrogen bonding, respectively, these specific interaction types may be discriminated from the benzene crystal (see also calculation details). Strikingly, these two interaction models that are commonly the only ones discussed for rationalizing molecular packing in benzamide were found to be both quantitatively and qualitatively insufficient to explain the preference of P1 over P3 type motifs. Indeed, in both benzene and formamide pseudo-crystals, molecular arrangement as corresponding to $\mathrm{P} 1$ is preferred, namely by 3.1 and $1.1 \mathrm{~kJ} \mathrm{~mol}^{-1}$ (per unit cell), respectively, thus overestimating the overall preference of P1 by a factor of two. However, much of the lattice energy arising from such intermolecular interactions is compensated by molecular torsion and valence angle deformation, where P1 is disfavoured over P3 by $1.5 \mathrm{~kJ} \mathrm{~mol}^{-1}$ (per unit cell). Surely, one may argue that the choice of proxy molecules imposes limitations to a quantitative analysis contrasting interactions with a $0.1 \mathrm{~kJ} \mathrm{~mol}^{-1}$ accuracy (i.e. $0.4 \mathrm{~kJ} \mathrm{~mol}^{-1}$ per unit cell and corresponding to a relative comparison, not absolute formation energies). However, to account for the 'missing' $(3.1+1.1-1.5)-1.9=0.8 \mathrm{~kJ} \mathrm{~mol}^{-1}$ per unit cell one should also consider further favouring of the P3 structure arising from an interaction type which has not been considered so far. Indeed, the latter may be easily deduced, as the proxy crystals account for benzene-benzene and amide-amide interactions, but (deliberately) ignore benzene-amide interactions.

As a consequence, we conclude that - at least qualitatively interactions of the polar amide groups with the benzene rings and possibly even longer ranged electrostatics are of importance to benzamide crystal polymorphism. This may be interpreted in terms of anion/cation- $\pi$ interactions, but because of the lack of reasonable proxies to mimic this interaction type on an exclusive basis akin to the above analysis, we can only deduce its energy indirectly. As all other types of molecular interactions were

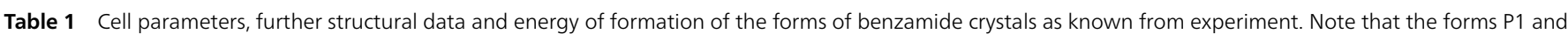

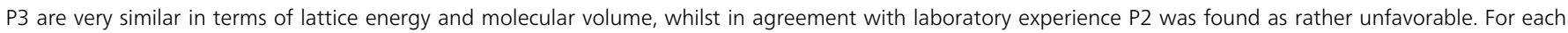

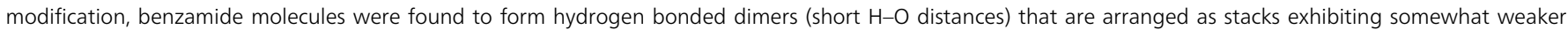
hydrogen bonding. Molecular deformation was characterized for each stack individually, resulting in two slightly different sets of torsion and valence angles

\begin{tabular}{|c|c|c|c|}
\hline & P1 (stable form) & $\mathrm{P} 2$ & P3 \\
\hline Number of molecules per unit cell & 4 & 8 & 4 \\
\hline$(a b c / \AA)$ & 5.437774 .9734220 .31571 & 17.8958014 .056445 .41586 & 4.976705 .2468221 .52106 \\
\hline$\left(\alpha \beta \gamma /^{\circ}\right)$ & 89.956786 .298590 .0858 & 90.000090 .005090 .0000 & 89.9695102 .429090 .2812 \\
\hline Volume per molecule & $137.07 \AA^{3}$ & $167.15 \AA^{3}$ & $137.20 \AA^{3}$ \\
\hline Torsion angle & $21.51^{\circ} / 21.58^{\circ}$ & $21.02^{\circ} / 26.09^{\circ}$ & $19.65^{\circ} / 20.20^{\circ}$ \\
\hline $\mathrm{N}-\mathrm{O}-\mathrm{C}$ angle & $154.07^{\circ} / 153.74^{\circ}$ & $164.88^{\circ} / 163.70^{\circ}$ & $153.88^{\circ} / 155.09^{\circ}$ \\
\hline H-bond length (dimers) & $1.81 \AA ̊ 81.81 \AA$ & $1.80 \AA / 1.81 \AA$ & 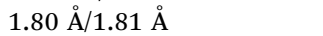 \\
\hline H-bond length (along stack) & $1.88 \AA / 1.87 \AA$ & $2.13 \AA / 2.13 \AA$ & $1.89 \AA ̊ 丿 1.88 \AA$ \\
\hline Lattice energy per molecule & $-117.6 \mathrm{~kJ} \mathrm{~mol}^{-1}$ & $P 1+21.7 \mathrm{~kJ} \mathrm{~mol}^{-1}$ & $\mathrm{P1}+0.5 \mathrm{~kJ} \mathrm{~mol}^{-1}$ \\
\hline
\end{tabular}


P1:

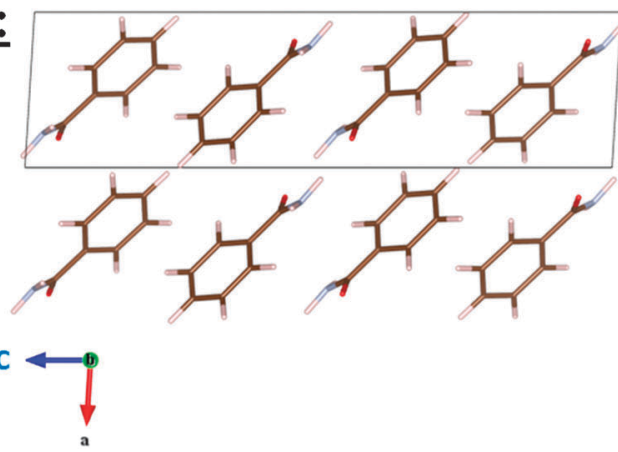

A
B
C

D

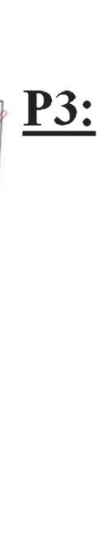

C

A

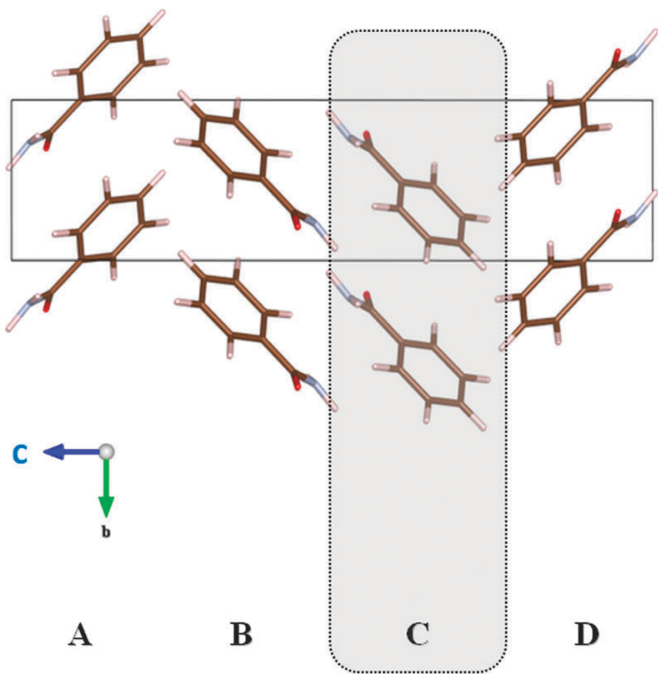

Fig. 1 P1 and P3 structure after energy optimization from DFT calculations, respectively. While the corresponding unit cells are indicated by rectangles, it is useful to discuss molecular packing in terms of staggered layers (shown for $C$, right) whose normal vector is aligned along the $c$ axis.

isolated, the benzene-amide interactions should account for favouring P3 motifs by $0.8 \mathrm{~kJ} \mathrm{~mol}^{-1}$ per unit cell, such that the overall balance results as the earlier mentioned $1.9 \mathrm{~kJ} \mathrm{~mol}^{-1}$ per unit cell preference of the P1 form of benzamide crystals. Thus, within the limitations of using proxy molecular crystals and adding up energy terms attributed to selected interaction types, we provide at least qualitative evidence for interaction types that favour P3 over P1 type motifs. This motivated us to search for pre-crystalline molecular arrangements of different P1 vs. P3 motifs enabling them to possibly act as precursors for a more directed polymorph synthesis.

From a structural point of view, both the P1 and P3 modifications may be characterized by hydrogen bonded layers of benzamide dimers which are staggered along the $c$ axis (Fig. 1). Along this line, the 4 molecules of the unit cell constitute 4 (001)-layers which are named A-D in the following. Hydrogen bonding in layers $\mathrm{A}$ and $\mathrm{D}$ is practically identical for both modifications. In the $\mathrm{B}$ and $\mathrm{C}$ layers, hydrogen bonding mainly differs by an orientation inversion, but not in terms of coordination numbers or strong differences of bond lengths. Thus, as the small difference in potential energy of forms P1 and P3 might suggest, hydrogen bonding is quite analogous in both polymorphs. As Fig. 1 suggests, the interactions of the benzene moieties account for the preference of P1 (Table 2).

This may be nicely demonstrated by contrasting the formation energies of isolated double layers. Cutting adjacent (001)-layers

Table 2 Potential energy of molecular packing in different motifs adopted from the P1 and P3 crystal structure (see also Fig. 1). The different tendencies observed for $A B / C D$ and $B C / D A$ double layers (see also Fig. 1) helps us to envisage nucleation routes to guide syntheses in favor of $\mathrm{P} 1$ and $\mathrm{P} 3$, respectively

\begin{tabular}{lllll}
\hline Formation energy per molecule/kJ mol & & P1 & P3 & $\begin{array}{l}\text { Difference } \\
(\mathrm{P} 1-\mathrm{P} 3)\end{array}$ \\
\hline A or B or C or D (single layer) & -61.33 & -61.89 & +0.56 \\
AB or CD (double layer) & -78.365 & -78.25 & -0.115 \\
BC or DA (double layer) & -98.955 & -99.31 & +0.355
\end{tabular}

from the P1 and P3 structures leads to two classes of double layers. The interface of $\mathrm{AB}$ and $\mathrm{CD}$ is characterized by benzene-benzene contacts, whilst $\mathrm{BC}$ and DA reflect the hydrogen bonded networks. Strikingly, the formation energies show different trends when contrasting molecular layers as cut from P1 and P3, respectively. While the $\mathrm{AB}$ and $\mathrm{CD}$ type double layers display a weak $\left(-0.2 \mathrm{~kJ} \mathrm{~mol}^{-1}\right.$ per dimer) favouring of the molecular arrangement as adopted from P1, for the BC and DA type layers the P1 type motifs are disfavoured by $+0.7 \mathrm{~kJ} \mathrm{~mol}^{-1}$ per dimer. Clearly, such isolated double layers cannot account for all aspects of lattice energy as in particular long range electrostatics are considered only incompletely. Nevertheless, we feel that our observations are of crucial relevance to the early stage of benzamide crystal nucleation as described in the following.

Whilst the hydrogen-bonded double layers $\mathrm{BC}$ and $\mathrm{DA}$ are energetically preferred with respect to their energy of formation from the vapour, in a polar solvent we expect the $\mathrm{AB}$ and $\mathrm{CD}$ layers to be favoured by hydrophobic segregation. Thus, by tuning the polarity of the solvent one might envision control of the driving force for benzamide dimerization and aggregation using the competition between benzamide-benzamide and (if applicable) benzamide-solvent hydrogen bonding. Combining this possibility given by the amphiphilic nature of benzamide with our findings related to the potential energy differences of P1 vs. P3 type motifs, it is intuitive to expect synthesis routes from aqueous solution to occur via hydrophobic segregation of the benzene moieties, leading to the $\mathrm{AB}(\mathrm{CD})$ type of benzene-benzene contacts during the initial stage of molecular association and eventual layer formation. Thus, such routes should lead to the P1 form of benzamide crystals. On the other hand, crystal nucleation from unpolar solvent (including nucleation from the vapour which we take as the ultimate unpolar solvent) should start from the formation of hydrogen-bonded benzamide dimers and aggregates. Thus, nucleation first leads to $\mathrm{BC}(\mathrm{DA})$ type layers before $\mathrm{AB}(\mathrm{CD})$ type layers are built. As a consequence, an unpolar (vapour) environment should promote P3 type motifs 
in infant benzamide aggregates. Despite obvious limitations arising from discussing layers instead of molecular clusters, the above concept can at least qualitatively explain experimental experiences. Indeed, precipitation from aqueous solution is known to lead to P1, whilst the preparation of $\mathrm{P} 3$ requires synthesis routes in much less polar solvent (benzene) or from the vapour. ${ }^{17,21}$

\section{Conclusions}

Dispersion-corrected density-functional theory calculations may provide molecular scale insights for rationalizing the competitions between structural motifs in molecular crystal polymorphs of benzamide. Our findings support established experimental evidence for the synthesis of benzamide forms P1 and $\mathrm{P} 3$ by relating different benzamide layer-layer interactions to different nucleation routes expected for the amphiphilic molecule when precipitated from polar or non-polar solvent. Apart from determining the role of specific structural motifs of the molecular crystal, contrasting benzene and amide pseudocrystals arranged at lattice sites and orientations corresponding to the benzamide crystal structure allows us to discriminate between different types of molecular interactions arising from a complex interplay of various contributions. Along these lines, we elaborated molecular arrangements with different tendencies to favour the P1 vs. P3 structure type, which are putative precursors to crystal nucleation of a desired polymorph.

\section{References}

1 D. E. Grigoriadis, et al., Neuropsychopharmacology, 2009, 34, 106-125.

2 C. Schön and M. Jansen, Angew. Chem., Ind. Eg. Engl., 1996, 108, 1358-1377 (Angew. Chem., Int. Ed. Engl., 1996, 35, 1286-1304).

3 R. S. Payne, R. J. Roberts, R. C. Rowe and R. Docherty, Int. J. Pharm., 1999, 177, 231.

4 R. C. B. Copley, S. A. Barnett, P. G. Karamertzanis, K. D. M. Harris, B. M. Kariuki, M. C. Xu, E. A. Nickels,
R. W. Lancaster and S. L. Price, Cryst. Growth Des., 2008, 8, 3474-3481.

5 M. Jansen, K. Doll and J. C. Schön, Acta Crystallogr., Sect. A: Fundam. Crystallogr., 2010, 66, 518-534.

6 S. L. Price, Acc. Chem. Res., 2009, 42(1), 117-126.

7 D. A. Bardwell, et al., Acta Crystallogr., Sect. B: Struct. Sci., 2011, 67, 535-551.

8 G. T. Beckham, B. Peters and B. L. Trout, J. Phys. Chem. B, 2008, 112, 7460-7466.

9 J. Anwar, S. C. Tuble and J. Kendrick, J. Am. Chem. Soc., 2007, 129(9), 2542-2547.

10 D. Benoit, P. Ectors, J. Breu and D. Zahn, Chem. Phys. Lett., 2011, 514, 274-277.

11 J. Anwar and D. Zahn, Angew. Chem., Int. Ed., 2011, 50, 1996-2013.

12 F. Wöhler and J. von Liebig, Ann. Pharmacother., 1832, 249-282.

13 P. Giannozzi, et al., J. Phys.: Condens. Matter, 2009, 21, 395502.

14 J. P. Perdew, K. Burke and M. Ernzerhof, Phys. Rev. Lett., 1996, 77(18), 3865-3868.

15 S. Grimme, J. Comput. Chem., 2006, 15, 1787-1799.

16 Q. Gao, G. A. Jeffrey, J. R. Ruble and R. K. McMullan, Acta Crystallogr., Sect. B: Struct. Sci., 1991, 47, 742-745.

17 J. Thun, L. Seyfarth, J. Senker, R. E. Dinnebier and J. Breu, Angew. Chem., Int. Ed., 2007, 46, 6729-6731.

18 W. I. F. David, K. Shankland, C. R. Pulham, N. Blagden, R. J. Davey and M. Song, Angew. Chem., Int. Ed., 2005, 44, 7032-7035; N. Blagden, R. Davey, G. Dent, M. Song, W. I. F. David, C. R. Pulham and K. Shankland, Cryst. Growth Des., 2005, 5, 2218-2224.

19 H. J. Monkhorst and J. D. Pack, Phys. Rev. B: Solid State, 1976, 13, 5188-5192.

20 J. Thun, M. Schöffel and J. Breu, Mol. Simul., 2008, 34, 1359-1370.

21 C. Butterhof, T. Martin, P. Ectors, D. Zahn, P. Niemietz, J. Senker, C. Näther and J. Breu, Cryst. Growth Des., 2012, 12(11), 5365-5372. 